\title{
Are alternative livelihood projects effective at reducing local threats to specified elements of biodiversity and/or improving or maintaining the conservation status of those elements?: a systematic review protocol
}

Dilys Roe ${ }^{1 *}$, Mike Day ${ }^{1,2}$, Francesca Booker $^{1,2}$, Wen Zhou ${ }^{2}$, Sophie Allebone-Webb ${ }^{3}$, Noëlle Kümpel ${ }^{3}$, Nicholas A O Hill ${ }^{3,7}$, Juliet Wright ${ }^{3,4}$, Niki Rust ${ }^{1,8}$, Terry CH Sunderland ${ }^{2}$, Kent Redford ${ }^{5}$ and Gillian Petrokofsky 2,6

\begin{abstract}
Background: Alternative livelihood projects are used by a variety of organisations as a tool for achieving conservation results. Yet these interventions, including their objectives, vary a great deal, and there is no single accepted definition of what constitutes an alternative livelihood project. In addition, very little is known about what impacts, if any, alternative livelihoods projects have had on biodiversity conservation, as well as what determines the success or failure of these interventions. Reflecting this concern, a resolution was passed at the IUCN World Conservation Congress in 2012 calling for a critical review of the benefits to biodiversity of alternative livelihood projects. This systematic review is intended to contribute to this resolution.

Methods/Design: This protocol details the methodology for exploring the research question: Are alternative livelihood projects effective at reducing local threats to specified elements of biodiversity and/or improving or maintaining the conservation status of those elements? The aim of this proposed systematic review is to provide an overview for researchers, policy makers and practitioners of the current state of the evidence base. To undertake this, the systematic review will explore peer-reviewed research from the bibliographic databases SCOPUS, Web of Science, CAB Abstracts, AGRIS and AGRICOLA using search terms and Boolean search operators. The systematic review will also explore grey literature sources by conducting searches on Google, subject specific websites and institutional websites. Additionally, the review will use calls for papers and snowballing techniques to further identify literature. In order to identify relevant evidence, inclusion criteria will be used to screen the titles and abstracts of the captured literature. Data will be extracted from the final list of relevant documents by using a questionnaire established through literature review and a stakeholder workshop. The key output of this review will be a report on the state of the evidence, and, if the data permits, an estimate of the effectiveness of alternative livelihood interventions.
\end{abstract}

Keywords: Alternative livelihoods, Biodiversity, Conservation, Community attitudes and conservation threats

\footnotetext{
* Correspondence: dilys.roe@iied.org

'International Institute for Environment and Development (IIED), 80-86 Grays Inn Road, London WC1X 8NH, UK

Full list of author information is available at the end of the article
} 


\section{Background}

Strategies designed to alleviate threats to biodiversity have been applied at a variety of scales from local to global. Recent attention has focused on global and regional scales due to concerns about the impacts of climate change and large-scale habitat loss. However, there has also been considerable investment in strategies designed for implementation at the local level. Many of these strategies have targeted the actions of people living in and around protected areas or other areas of biodiversity importance, and have consisted of both negative and positive incentives to change people's attitudes and behaviour. These incentives are intended to reduce or cease people's exploitation of components of biodiversity that are of conservation concern. Commonly-used disincentives include resource access restrictions, with penalties imposed if restrictions are not adhered to; increased law enforcement; and land/resource use zoning. Frequently-used positive incentives include payments for ecosystem services and other compensatory mechanisms, and so-called "alternative livelihoods projects", which are the focus of this review.

Alternative livelihood projects are poorly defined in the literature - indeed there is no common definition. In general, however, they can be understood to be an approach to achieving biodiversity conservation by substituting a livelihood strategy that is causing harm to a biodiversity target (for example, through unsustainable use), for one that has a more positive impact. In other words, they seek to reduce locally-driven threats to biodiversity. In some cases this might mean providing an alternative resource to the one that is being exploited. For example, encouraging local people to farm cane rats as a source of protein, rather than hunting bushmeat (e.g. Development d'Alternatives au Bracconage en Afrique Centrale project [1]).

In other cases, the focus of the project may be on providing an alternative occupation or source of income so as to reduce the need to exploit the biodiversity target. Commonly used alternative occupations include craft making [2], bee-keeping [3] or butterfly farming [4] as a substitute for expanding subsistence agriculture around protected areas, or seaweed farming as an alternative to artisanal fishing [5,6]. Finally, a third approach involves encouraging an alternative method of exploiting a resource that has a lower impact than the original method. Examples of such interventions include the promotion of fuel-efficient stoves to reduce the demand for firewood [7]; or the introduction of a mariculture programme to ameliorate pressure on a certain fish species [8].

Alternative livelihoods projects are sometimes standalone initiatives and at other times part of a broader integrated conservation and development (ICD) programme. In all cases, the alternative livelihoods projects or components share a common objective: "to provide an alternative means of making a living that reduces pressure on exploited resources" [3]. The use of alternative livelihood projects is not limited to the conservation sector. In Afghanistan, the promotion of alternative livelihoods is a central pillar of the government's counter narcotics strategy [9] while in Ghana, alternative livelihoods projects such as cassava farming, poultry farming, and grass-cutter or snail rearing have been promoted in order to combat illegal artisanal mining [10].

The on-going commitment to alternative livelihood approaches within the conservation sector is demonstrated by the United States Agency for International Development (USAID) - funded Central African Regional Program for the Environment (CARPE). This initiative includes an objective to "expand alternative livelihood opportunities for rural communities" as a goal in its latest (2012-2020) phase [11]. Yet despite such investment, very little is known about what has worked, what has not worked and why. Furthermore, both conservation and development practitioners have expressed concern that the alternative livelihoods approach may be flawed. In particular, with the emergence of the sustainable livelihoods framework in the late 1990s [12], it is better recognised that poor households are likely to engage in a combination of diverse livelihood activities [13,14]. Commentators have suggested, therefore, that because of this multiplicity of activities the introduction of an alternative livelihood activity may simply be incorporated into the overall mix of activities rather than acting as a genuine replacement [15].

Reflecting this concern, a motion was passed at the IUCN World Conservation Congress in 2012 calling for a critical review of biodiversity benefits of alternative livelihood projects and the development of "best practice guidelines for alternative livelihood projects to ensure sustainable benefits to species, ecosystems and people" [16]. This systematic review is a response to that call.

\section{Objective of the review}

The objective of this systematic review is to critically examine the evidence on the effectiveness of alternative livelihoods projects in order to inform the future design of such interventions. The goal is to conduct an overview of the state of the evidence base and illuminate the strengths, weaknesses and prevailing knowledge gaps. This review arose from a meeting convened by the Zoological Society of London (ZSL) in May 2012, which challenged a small group of participants to identify case studies of alternative livelihoods interventions that have experienced success, specifically in achieving positive conservation outcomes. This in turn stimulated passing of the aforementioned resolution to IUCN calling for a critical review in order to help guide future donor investments. 
The timing of the IUCN resolution coincided with a move by the Center for International Forestry Research (CIFOR) to promote evidence-based approaches to science-policy dialogue. As part of this, CIFOR established an "Evidence-Based Forestry Initiative" to act as a platform for promoting systematic reviews of key policy questions, including this review [15]. CIFOR, the Institute for International Environment and Development (IIED) and ZSL are collaborating on this review and form the core review team.

The primary research question for the review (summarised in Table 1) is: "Are alternative livelihood projects effective at reducing local threats to specified elements of biodiversity and/or improving or maintaining the conservation status of those elements?" Within this, 'alternative livelihood projects" are defined as interventions that "seek to alleviate a human threat to biodiversity through providing, or encouraging the use of an alternative resource; an alternative occupation; or an alternative method (lower impact) of exploitation"; and "effectiveness is defined as: 1) threat alleviation through changes in human attitude, behaviour and/or wellbeing; and ultimately 2) improvements in the conservation status of the specified element of biodiversity under threat. The secondary question is: What is the state of the evidence base for the effectiveness of alternative livelihood projects for biodiversity conservation?

Our definition of alternative livelihoods projects distinguishes the review's scope from having a broader focus on 'livelihood projects', which can include any conservation intervention that addresses the economic needs of local people. This includes, for example, community-based conservation, payments for ecosystem service (PES) schemes and integrated conservation and development programmes (although we would include such approaches if they include an alternative livelihoods intervention as a component of a wider scheme). Specifically, our definition implies that there is some kind of substitution of an unsustainable activity for one that is considered as sustainable - or even has a positive effect -on the conservation status of a particular biodiversity target (for example species, group of species, habitat).

\section{Methods/Design}

Literature searching

Developing and testing a search strategy

Initial literature searching was tested in Elsevier's Scopus

[17] and Thomson Reuter's Web of Knowledge (Science)
[18]. A list of search terms was compiled for the population and intervention elements that construct the research question. Search terms included synonyms for alternative livelihoods, examples of specific types of alternative livelihoods interventions (such as beekeeping or cane rat farming) and terms related to biodiversity or conservation. This list was developed iteratively as more terms were identified from the literature. Final search terms were selected based on the number of search results they generated and the presence of publications from a test library of 15 relevant studies. This is an evolving test library with further relevant papers added as they are identified to pass our primary inclusion criteria (see 'Additional file 1: A list of Search Terms used and the Test Library').

A scoping search was also conducted in CABI's database CAB Abstracts [19] using the OvidSP platform [20]. This search used the CAB Abstracts Thesaurus to identify search terms and generated 12,389 search results (see 'Additional file 2: The Search Strategy used for Bibliographic Databases'). The search was intended to be broad to allow for an initial review of the literature, paying attention to the terminology used to refer to alternative livelihoods in the titles and abstracts. It is expected that this process will help to further refine the search strategy generated from testing Scopus and Web of Knowledge.

In addition to the bibliographic databases, search terms were tested in the search engine platform Google Scholar [21]. The terms that produced relevant hits were combined to produce six search strings of alternative livelihood synonyms and eight search strings for specific types of alternative livelihoods interventions (see 'Additional file 3: The Search Strategy used for Internet Search Engines').

\section{Search terms and languages}

Search terms are detailed in 'Additional file 1: A list of Search Terms used and the Test Library'. Searches will be carried out using the listed English terms in the first instance, and replicated in French and Spanish. Although, this will not be necessary for the bibliographic searches as titles and abstracts for all foreign language papers are included in English in the selected databases. The team's linguistic abilities and time limitations preclude any further language coverage. Terms listed in three relevant multilingual subject-specialist thesauri will also be incorporated: AGROVOC [22], CAB Thesaurus (English and Spanish terms) [23] and NAL Thesaurus [24].

Table 1 Elements of the systematic review question

\begin{tabular}{llll}
\hline Population & Intervention & Comparators & Outcomes \\
\hline $\begin{array}{l}\text { Biodiversity } \\
\text { target }\end{array}$ & $\begin{array}{l}\text { Alternative livelihoods } \\
\text { project }\end{array}$ & $\begin{array}{l}\text { Prior to alternative livelihoods project intervention/ } \\
\text { without alternative livelihood intervention }\end{array}$ & $\begin{array}{l}\text { Reduced threat and/or improved conservation } \\
\text { status of specified biodiversity target }\end{array}$ \\
\hline
\end{tabular}


The generic search strategy is shown in more detail across Additional files 1, 2, 3 and 4. The strategy will vary slightly between each bibliographic database according to their particular field structures use of Boolean operators, wildcards, proximity searches and the exclusion of irrelevant subject areas; full descriptions of the final search strategies will be documented.

\section{Sources of publications}

\section{(a) Bibliographic databases}

The following bibliographic databases will be searched for publications:

- Scopus [17]

- Web of Science [18]

- CAB Abstracts [19]

- AGRIS [25]

- AGRICOLA [26]

\section{(b) Individual journals}

A number of individual journals will be targeted. The Directory of Open Access Journals (DOAJ) [27] will be used to identify freely accessible publications in as well as INASP's (International Network for the Availability of Scientific Publications) [28] regional journal platforms such as African Journals Online (AJOL) [29], Asia Journals Online [30] and the Poverty and Conservation Learning Group (PCLG) journal digest [31]. For searches in all these platforms, simple search terms will be used as follows: conservation AND development, alternative livelihoods, or just livelihood where characters are limited. The equivalent French and Spanish terms will also be used where appropriate.

\section{(c) Internet searching}

Google Scholar [21] will be searched using a total of 14 search strings. The first 150 search results, when organised by relevance, will be captured for the 6 alternative livelihood synonym strings, while the first 100 results will be captured for those 8 strings using the specific alternative livelihood intervention terms. This is because the latter 8 strings give a narrower range of results when compared with the 6 strings that use broader alternative livelihood synonyms. These Google Scholar strings will also be translated into French and Spanish languages and the results captured. Searches for grey literature will also be conducted using simple search terms in the search engine Google [32]. The first 100 results, when ordered by relevance, will be reviewed (see 'Additional file 3: The Search Strategy used for Internet Search Engines').

\section{(d) Specialist searching}

Grey literature will be searched through the websites of key donor, implementer and research organisations. 'Additional file 4: The Search Strategy to be used for
Specialist Websites' details the list of organisations that will be targeted. These organisations have been selected according to their prominence in conservation and development, as well as the ability to search their websites. A number of subject specific websites with potential relevant publications will also be searched. Searches on these websites will use the simple search terms that were outlined in the individual journals section (e) above.

\section{(e) Theses and dissertation repositories}

Six theses and dissertation repositories will be targeted; Ethos [33], DART-Europe E thesis [34], National ETD Portal South Africa [35], Open Access Theses and Dissertations [36] and ProQuest Digital Dissertations and Theses [37]. Again, searches on these repositories will use the simple search terms that were outlined in the individual journals section (e) above.

\section{(f) Stakeholder engagement}

Through a process of stakeholder engagement, expert advice has been, and will continue to be, sought on relevant key papers from both the peer reviewed and grey literature. On the $18^{\text {th }}$ December 2013, a short presentation on the project was provided to the UK Bushmeat Working Group, and feedback on the proposed approach and on relevant papers requested. The project has been summarised in a flyer to be disseminated via the project partners' websites and at relevant conferences and workshops attended by team members.

In addition, a call for case study examples of alternative livelihood projects and key papers that measure such intervention's effectiveness for achieving biodiversity conservation has been issued on relevant email listservs including: the Biodiversity-L Listserv, a peer to peer announcement list run by the International Institute for Sustainable Development [38]; and the Poverty and Conservation Learning Group (PCLG), an international network of conservation and development organisations coordinated by IIED [32]. A blog [39] has also been produced and promoted via social networks, to encourage the sharing of relevant case studies and key papers.

Further efforts will include requesting our Advisory Board of experts working within the field of conservation and development, who will guide the systematic review, to list five to ten key publications that are relevant to the research question.

The references of all documents recommended will be screened for relevance (see Study inclusion criteria), as well as the references of those documents subsequently included through this process, in a 'backward snowball' [40].

\section{Estimating the comprehensiveness of the search}

The iterative development of search terms - and their piloting against a test library of key papers - will ensure the search is as comprehensive as possible. In addition, searching in both French and Spanish languages will 
broaden the coverage of the literature captured. Potential limitations to the review's comprehensiveness include the lack of standard terminology to describe 'alternative livelihoods projects'. As a result, it could be that many programmes that fall into the definition used by this systematic review for 'alternative livelihoods projects' are not captured. The iterative development of search terms will help to minimise the potential biases caused by this limitation.

A further limitation is the extent to which grey literature can be accessed. It is anticipated that much of what is known about "alternative livelihood projects" may not be documented in scientific literature but rather in project records. This protocol outlines an extensive methodology that includes engaging with individuals and organisations to help in the process of identification and examination of as many repositories of information as possible. However, access to documents may be restricted in many cases.

\section{Study inclusion criteria}

After all the literature captured through searches is gathered, and duplicates are removed, the inclusion criteria detailed in Table 2 will be applied to each document. A Kappa analysis will be performed on a random (minimum of 100) sample of the titles and abstracts to measure the level of agreement between the five researchers in applying these criteria [41,42]. If the kappa is lower than 0.6, reviewers will discuss the discrepancies and clarify any differences in the interpretation of the inclusion criteria. The five reviewers involved in this process have a role in screening the titles, abstracts, and full texts.

When applying the inclusion criteria to the search results, documents will be assessed independently, with each reviewer assigned to a proportion of the literature. A reviewer will be able to include, exclude or query a document on the basis of its title, followed by its abstract. Where there is doubt, a reviewer will be encouraged to query the inclusion of a document with the other reviewers during weekly team meetings. Exclusion at this stage will be conservative, ensuring that if there is any doubt regarding a document's relevance it is retained until an assessment based on the full text.

Once documents have been included or excluded on the basis of their titles or abstracts, all reasonable effort will be made to obtain the full text electronic or paper

\section{Table 2 Primary inclusion criteria 1}

Articles will be included 1. Relevant population: The study assesses in the review if they an element of biodiversity (for example meet these criteria. species, group of species, habitat).

2. Relevant intervention: The study involves an intervention that aims to achieve conservation of biodiversity through a livelihoods intervention. copies of the remaining documents. This includes emailing corresponding authors where the full text is not available to download from web-based sources. At this point, any documents that the review team is uncertain about will be screened according to their full text.

\section{Potential effect modifiers and reasons for heterogeneity}

There are a number of effect modifiers that the review team will consider including: ecosystem/habitat type, size and location; protection status including time since establishment; management type; presence of other conservation mechanisms or associated development projects; availability \& access of other natural resources; country (including economic indices); population density; baseline poverty; livelihood strategies.

\section{Study quality assessment}

To minimize study bias and ensure validity of the studies with respect to the review question, we will use a checklist approach to assess the quality of included studies. The basic quality standard that we will apply is that studies must have included a measure of effectiveness in their analysis. Any studies that do not include a clearly articulated measure of effectiveness will be excluded from the synthesis. Beyond this basic standard we will also check for the following:

- Clear and repeatable methodology

- Methodology appropriate to the assessment of effectiveness

- Methodology allows for causal links to be established

- Reported findings consistent with methodology employed

- Sample size is specified and appropriate

- Confounding factors are taken into account

Studies which meet none of the checklist quality criteria will not be included in the synthesis. Our synthesis will then distinguish between studies which meet a high quality score (meets all the criteria on the checklist), those with a low score ( 2 or less criteria me) and those in between (medium quality score). The checklist criteria will be tested on key references and will be further refined during the data extraction process, including through consultation with the Advisory Group.

\section{Data extraction strategy}

We will use a short data extraction template, developed iteratively with stakeholders (modelled on Isasi-Catalá, 2009 [43]; itself adapted from Pullin \& Knight 2001 [44]) to capture relevant data that will be analysed in the review. The data extraction strategy will be piloted using the 15 articles in the test library. 
Data to be extracted will include:

1. Bibliographic information: author, year, title, publication, place published, publisher.

2. Basic information: location of study, alternative livelihood intervention (alternative resource/method/ occupation), date \& duration of the intervention, stand-alone intervention or component of a wider project, project funder(s) \& implementer(s).

3. Details of evidence type: source, location of primary author, study design, methodology, type of data, unit of analysis, duration of study.

4. Relevant detail considered by study: conservation target, target group of the alternative livelihood intervention, scale of the alternative livelihood intervention, and conceptual link between alternative livelihood intervention \& conservation intention.

As part of the data extraction, articles will be assessed to determine if they have included some assessment of the effectiveness of the alternative livelihoods intervention. Those that have done so will be subjected to second phase of data extraction using a second similarlyderived template. Data to be extracted in this phase will include:

5. Details of outcomes: effectiveness measure used (attitudinal/behavioural/change in conservation status etc.), reported effect (positive/negative/no effect), duration of impacts, scale \& sustainability of impacts, and the nature of any secondary impacts.

The data extraction templates will be designed so that wherever possible fixed answers will be selected from a coded, drop down list. Entering the answers into the spreadsheet in numerical code format will then allow for subsequent analysis with descriptive statistics. Where pre-determined, coded options are not possible, qualitative narrative data will be collected.

\section{Data management}

The final results from the searches outlined in 3.1.3 will be added to a Zotero library [45]. Microsoft Excel [46] will be used to collect the results of the data extraction.

\section{Data synthesis and presentation}

For the overall description of the state of the evidence base - the systematic map - we plan to provide a narrative mapping of all the articles that were reviewed in full. We will use descriptive statistics to summarise trends, to map the quantity and type of articles reviewed, and to highlight their relevance to the primary question. Table 3 outlines the main categories of the data that will be analysed through producing summary graphs and charts to provide an overview of the evidence base. The map will be provided in a narrative and graphic form (using charts and tables to illustrate the results found) and we will also explore the potential for recording data on a project-byproject basis in an Access - or custom-built - database that can subsequently be made available to other researchers and practitioners online.

For the more detailed analysis of the studies that meet our quality assessment criteria for the systematic review, we will again predominantly rely upon descriptive statistics and narrative synthesis. We will explore the possibility of meta-analysis in order to quantify and synthesise the overall findings. However, given the variation in types of interventions that the scoping study revealed we anticipate that meta-analysis is not likely to be feasible. Our

Table 3 Categories of data to be included in the data synthesis

\begin{tabular}{|c|c|}
\hline \multirow[t]{3}{*}{ Nature of evidence } & - Sources of evidence (journal types and subjects, grey literature) \\
\hline & - Types of evidence (study design, data sources, scale of analysis) \\
\hline & - Producers of evidence (location of primary authors/institutions) \\
\hline \multirow{6}{*}{$\begin{array}{l}\text { Representativeness and } \\
\text { coverage of evidence }\end{array}$} & - Geographic coverage (countries, regions) \\
\hline & • Ecological coverage (biomes, habitats) \\
\hline & - Components/attributes of biodiversity studied (species, group of species) \\
\hline & - Types of alternative livelihood projects (i.e. alternative resource, alterative occupation or alternative method) \\
\hline & - Measure of effectiveness used \\
\hline & - Nature of impacts reported (positive, negative, neutral) and relative proportions of each type \\
\hline \multirow[t]{3}{*}{ Measure of effectiveness } & - Scale of impacts and numbers of studies that address scale \\
\hline & - Nature of any secondary impacts (including social \& economic) \& evidence of any trade offs or synergies. \\
\hline & - Sustainability of impacts - no's of studies that consider sustainability issues \\
\hline $\begin{array}{l}\text { Policy, institutions and } \\
\text { governance }\end{array}$ & - Attention paid to underlying policy, institutional and governance issues \\
\hline
\end{tabular}


synthesis will include details of the comparative effectiveness of different alternative livelihood project approaches (alternative resource, alternative occupation, alternative method); the most common outcomes (changes in attitude compared to changes in behavior or changes in conservation status); and - if possible - the factors limiting effectiveness (access to markets; type and level of threat; implementation issues). In all cases, as mentioned above, we will distinguish between studies of different quality in our synthesis. The data extraction strategy and synthesis approach will be further refined during the review process, including through consultation with the Advisory Group.

\section{Additional files}

Additional file 1: A List of Search Terms used and the Test Library.
Additional file 2: The Search Strategy used for Bibliographic
Databases.
Additional file 3: The Search Strategy used for Internet Search
Engines.
Additional file 4 The Search Strategy to be used for Specialist
Websites.

\section{Competing interests}

The authors declare that they have no competing interests.

\section{Authors' contributions}

DR, MD \& FB co-drafted the manuscript, while SAW, WZ, NW, NH, JW, GP, TS, KR provided comments. FB, MD and WZ developed the search strategy, with input from GP. DR, MD, FB \& GP jointly developed the data extraction strategy. DR will coordinate the review including the analysis and presentation of the results with help from MD, FB, WZ, SAW \& GP. All authors read and approved of the final manuscript.

\section{Acknowledgements}

The authors would like to thank Professor Peter Kanowski and Dr Peter Holmgren of the Centre for International Forestry Research for their support for the evidence-based forestry initiative, which is funded by a grant to CIFOR from the UK's Department for International Development (DfID). We would also like to thank staff of the Bodleian library, University of Oxford for help locating sources of evidence.

This work has been conducted with the financial assistance of the UK Department for International Development, through its KNOW-FOR program grant to CIFOR, and funding partners who have contributed to the CGIAR Fund.

\section{Author details}

${ }^{1}$ International Institute for Environment and Development (IIED), 80-86 Grays Inn Road, London WC1X 8NH, UK. ${ }^{2}$ Centre for International Forestry Research (CIFOR), Jalan CIFOR, Situ Gede, Sindang Barang, Bogor 16115, Indonesia. ${ }^{3}$ Zoological Society of London (ZSL), Regent's Park, London NW1 4RY, UK. ${ }^{4}$ Department of Life Sciences, Imperial College London, Silwood Park Campus, Buckhurst Road, Ascot SL5 7PY, UK. ${ }^{5}$ Archipelago Consulting, P.O. Box 4750, Portland, Maine 04112-4750, USA. ${ }^{6}$ Department of Zoology, University of Oxford, The Tinbergen Building, South Parks Road, Oxford OX1 3PS, UK. 'Conservation Science Group, Department of Zoology, University of Cambridge, Cambridge CB2 3EJ, UK. ${ }^{8}$ Durrell Institute of Conservation and Ecology, University of Kent, Canterbury, Kent CT2 7NZ, UK.

Received: 9 December 2013 Accepted: 11 March 2014 Published: 25 March 2014

\section{References}

1. Secretariat of the Convention on Biological Diversity (SCBD): Livelihood alternatives for the unsustainable use of bushmeat. In Report prepared for the CBD Bushmeat Liaison Group. Technical Series No. 60. Montreal: SCBD; 2011:46.

2. Triet $\mathrm{R}$ : Combining biodiversity conservation with poverty alleviation - a case study in the Mekong Delta Vietnam. Aquatic Ecosystem Health Manage 2010, 13:41-46.

3. Munthali S, Mughogho D: Economic incentives for conservation: beekeeping and saturniidae caterpillar utilization by rural communities. Biodivers Conserv 1992, 1:142-154.

4. Gordan I, Ayiemba W: Harnessing butterfly biodiversity for improving livelihoods and forest conservation: The Kipepeo Project. J Environ Dev 2003, 12:82-98.

5. Sievanen L, Crawford B, Pollnac R, Lowe C: Weeding through assumptions of livelihood approaches in ICM: seaweed farming in the Philippines and Indonesia. Ocean Coast Manag 2005, 48:297-313.

6. Hill N, Rowcliffe M, Koldewy H, Milner-Gulland EJ: The Interaction between Seaweed Farming as an Alternative Occupation and Fisher Numbers in the Central Philippines. Conserv Biol 2011, 2:324-334.

7. DeWan A, Green K, Li X, Hayden D: Using social marketing tools to increase fuel-efficient adoption for conservation of the golden snubnosed monkey, Gansu Province, China. Conserv Evid 2013, 10:32-36.

8. Lui M, Sadovy de Mitcheson Y: Profile of a fishery collapse: why mariculture failed to save the large yellow croaker. Fish Fish 2008, 2008:219-242.

9. Mansfield D, Pain A: Alternative Livelihoods: substance or Slogan?. Kabul, Afghanistan: Afghanistan Research and Evaluation Unit (AREA) Briefing Paper; 2005. http://ageconsearch.umn.edu/bitstream/14650/1/bp05ma01. pdf.

10. Hilson G, Banchirigah SM: Are alternative livelihood projects alleviating in poverty in mining communities? Experiences from Ghana. J Dev Stud 2009, 45:172-196.

11. U.S. Agency for International Development (US AID)/Central Africa Regional Program for the Environment (CARPE): Regional Development Cooperation Strategy 2012-2020. 1-38 [http://www.usaid.gov/sites/ default/files/documents/1860/CARPE_RDCS_0.pdf]

12. Carney D: Sustainable Rural Livelihoods: What contribution can we make? In Department for International Development's Natural Advisers' Conference, July 1998. London: Department for International Development (DfID); 1998

13. Department for International Development (DFID): Sustainable livelihoods guidance sheets numbers 1-8. 1999a, 2000d, 2001. [www.livelihoods.org]

14. Department for International Development (DFID): Sustainable Livelihoods and Poverty Elimination. London: Department for International Development (DfID); 1999b.

15. Brown K: Three challenges for a real people-centred conservation. Glob Ecol Biogeogr 2003, 12:89-92.

16. International Union for the Conservation of Nature (IUCN): Motion 145. In World Conservation Congress, 6-15 $5^{\text {th }}$ September 2012; Jeju, Korea. 2012. Access online: [https://portals.iucn.org/docs/2012congress/motions/en/M-145-2012EN.pdf]

17. Scopus. [http://www.scopus.com/]

18. Web of knowledge. [http://wok.mimas.ac.uk/]

19. Cab abstracts. [http://www.cabi.org/]

20. OvidSP. [https://ovidsp.ovid.com/]

21. Google scholar. [http://scholar.google.co.uk/]

22. Cab thesaurus. [http://www.cabi.org/cabthesaurus/]

23. AGROVOC. [http://aims.fao.org/standards/agrovoc/]

24. NAL thesaurus. [http://agclass.nal.usda.gov/]

25. AGRIS. [http://agris.fao.org/agris-search/index.do]

26. AGRICOLA. [http://agricola.nal.usda.gov/]

27. Directory of Open Access Journals (DOAJ). [http://www.doaj.org/]

28. International Network for the Availability of Scientific Publications (INASP). [http://www.inasp.info/]

29. African Journals Online (AJOL). [http://www.ajol.info/]

30. Asian Journals Online. [http://ajouronline.com/]

31. Poverty and Conservation Learning Group (PCLG). [http://povertyand conservation.info/

32. Google. [https://www.google.com/]

33. British Library Electronic Theses Online Service (EThOS). [http://ethos.bl. uk/Home.do]

34. DART Europe E- thesis Portal. [http://www.dart-europe.eu/basic-search. php] 
35. National ETD Portal of South Africa. [http://www.netd.ac.za/]

36. Open Access Theses and Dissertations. [http://oatd.org/]

37. ProQuest. [http://www.proquest.co.uk/en-UK]

38. Biodiversity-L Listserve. [http://www.iisd.ca/email/biodiversity-L.htm]

39. Day M: Are 'alternative livelihoods' projects effective? 2014 [http://www.iied.org/are-alternative-livelihoods-projects-effective]

40. Hagen-Zanker J, Mallet R: How to do a rigorous, evidence-focused literature review in international development, A Guidance Note. Overseas Development Institute; 2013 [http://www.odi.org.uk/sites/odi.org.uk/files/odi-assets/ publications-opinion-files/8572.pdf]

41. Cohen J: A coefficient of agreement for nominal scales. Educ Psychol Meas 1960, 20:37-46.

42. Edwards P, Clarke M, DiGuiseppi C, Pratap S, Roberts I, Wentz R: Identification of randomized controlled trials in systematic reviews: accuracy and reliability of screening records. Stat Med 2002, 21:1635-1640.

43. Isasi-Catalá E: Is translocation of problematic jaguars (Panthera onca) an effective strategy to resolve human-predator conflicts? 2009. CEE protocol 08-018 (SR55). Collaboration for Environmental Evidence. http://www.environmentalevidence.org/Documents/Final_protocols/ Protocol55.pdf.

44. Pullin A, Knight T: Effectiveness in conservation practice: pointers from Medicine and Public Health. Conserv Biol 2001, 15:50-54.

45. Zotero. [http://www.zotero.org/]

46. Microsoft Excel. [http://office.microsoft.com/en-gb/excel/]

doi:10.1186/2047-2382-3-6

Cite this article as: Roe et al:: Are alternative livelihood projects effective at reducing local threats to specified elements of biodiversity and/or improving or maintaining the conservation status of those elements?:

a systematic review protocol. Environmental Evidence 2014 3:6.

\section{Submit your next manuscript to BioMed Central and take full advantage of:}

- Convenient online submission

- Thorough peer review

- No space constraints or color figure charges

- Immediate publication on acceptance

- Inclusion in PubMed, CAS, Scopus and Google Scholar

- Research which is freely available for redistribution 